\title{
Contrast-enhanced Ocular Ultrasonography in Brachycephalic Dogs
}

\author{
Thais Guimarães Morato Abreu', Marcus Antônio Rossi Feliciano², Marjury Cristina Maronezi', \\ Ricardo Andres Ramirez Uscategui', Gabriela Morais Madruga', Karina Kamachi Kobashigawa', \\ Roberta Martins Crivelaro', Roberto Thiesen ${ }^{3}$ \& José Luiz Laus'
}

\begin{abstract}
Background: Contrast Enchantment Ultrasonography (CEUS) allows for detection of vascular flows that are difficult to detect with traditional methods. There were no reports found in the literature of the use of CEUS to evaluate the vascular pattern of the eye of brachycephalic dogs. The objective of this paper is to describe ultrasound findings observed in the eyes of healthy brachycephalic dogs subjected to CEUS.

Materials, Methods \& Results: Thirty adult brachycephalic dogs were subjected to physical, laboratorial, and ophthalmic examination. The eye structures were evaluated using CEUS after intravenous administration of Sonovue ${ }^{\circledast}$. Contrast enhancement was seen in 52 eyes with a homogeneous and centrifugal filling pattern. Structures adequately enhanced consisted of the optic nerve, the retina-choroid-sclera complex (RCSC), and the ciliary body. The optic nerve was hyperenhanced and the other structures were isoenhanced. The wash-in time, peak enhancement time, and wash-out time were similar for both right and left eyes. Mean values and standard deviation were calculated for contrast wash-in time, peak enhancement time, and wash-out time in the optic nerve, RCSC, and ciliary body.

Discussion: Ultrasound is an important diagnostic resource for veterinary ophthalmology and provides relevant information for the detection of pathological conditions of the eyes of animals. The contrast-enhanced ultrasound allows evaluation of tissues at the capillary level (macro and microcirculation), complementing the vascular ultrasound scan. This study brings original information on contrast-enhanced ultrasound findings for evaluation of eyes in dogs, creating novel diagnostic possibilities for the use of this imaging technique in veterinary ophthalmology. The vascular pattern identified in this study, with enhancement starting at the region of the optic nerve and retina, and then ciliary body (with the optic nerve as the most hyperenhanced structure) is consistent with the expected pattern for dogs without ophthalmic disorders. The posterior ciliary artery is primarily responsible for supplying the optic nerve, later irrigating the retina, lateral and medial segments of the ciliary body. Some works on the evaluation of orbital vascular pattern in humans report a significant variability on anatomical course of the ophthalmic artery and its branches. However, in our study, no significant variation was observed on vascular flow of dogs of different brachycephalic breeds. Retinal blood vessels consist of arteries and veins that emerge from the optic nerve region and from the choroid. Contrast enhancement at the region of the RCSC is justified by the presence of vessels from the choroid that are irrigated by posterior ciliary arteries and drained by vorticose veins, since this structure is responsible for the nutrition of the external portion of the retina. Wash-in, peak enhancement, and wash-out times were similar for right and left eyes owing to anatomical and physiological similarities in vascularization. The homogeneous contrast-filling pattern is of paramount importance, since it will allow differentiation of abnormalities in sick animals, such as partial or total loss of capillarization, which will cause heterogeneity or absence of capillary fill. It will also be important for early diagnosis and evaluation of vascular alterations, especially ischemic changes, especially glaucoma, intraocular neoplasm, progressive retina atrophy, and uveitis. CEUS gives relevant information about the vascular pattern of the eyes of brachycephalic dogs, and is a quick, non-invasive, and safe exam.
\end{abstract}

Keywords: ciliary body, dog, ophthalmology,optic nerve, retina, vascularization.

DOI: $10.22456 / 1679-9216.81808$

${ }^{1}$ Departamento de Clínica e Cirurgia Veterinária, Faculdade de Ciências Agrárias e Veterinárias - UNESP, Jaboticabal, SP. ${ }^{2}$ Departamento de Reprodução Animal, Departamento de Medicina Veterinária Preventiva e Reprodução Animal, Faculdade de Ciências Agrárias e Veterinárias - UNESP, Jaboticabal, SP. ${ }^{3}$ Departamento de Medicina Veterinária, UNIPAMPA, Uruguaiana, RS, Brazil. CORRESPONDENCE: T.G.M. Abreu [thaismorato@ yahoo.com.br - Tel.: +55 (35) 992393516]. FCAV - UNESP. Via de Acesso Prof. Dr. Paulo Donato Castellani s/n. Bairro Distrito Industrial. CEP 14.884-900 Jaboticabal, SP, Brazil. 


\section{INTRODUCTION}

Microbubbles are a type of contrast-enhanced ultrasonography (CEUS). CEUS is a recent imaging technique that uses highly reflective structures capable of increasing the spectrum of Doppler signs. Microbubbles have diameters of 1 to $6 \mathrm{~mm}$, which enables passage through capillary beds and remain strictly intravascular, with no interstitial diffusion or urinary excretion, and its use is safe and free from hemodynamic effects [16].

In veterinary medicine, ultrasound using microbubbles contrast has been used for evaluation of spleen in dogs [20,24], hepatic carcinoma [2], and evaluation of renal [11], testicular and prostatic lesions [26].

Recently, this contrast technique has been used in human ophthalmology yielding important results in the evaluation of therapeutic response in patients with uveal melanoma subjected to radiosurgery [25]. Otherwise other studies reported the tissue vascularization after insertion of hydroxyapatite orbital implants [27]. Another reported investigated the vascular pattern in patients with choroidal melanoma, thus helping with the early diagnosis of this disease [17,29] and in studies of murine models of choroidal melanoma [28].

Considering the scarcity of studies that describe the image characteristics and applicability of this contrast-enhanced ultrasound technique for evaluation of the eye in dogs, the present study had the objective of describing ultrasound findings observed in the eye of healthy brachycephalic dogs subjected to CEUS.

\section{MATERIALS AND METHODS}

Animals

This study was approved by the Animal Ethic and Welfare Committee (Univ Estadual Paulista) protocol $\mathrm{N}^{\circ} 9516 / 15$. This prospective study included 84 eyes of 42 brachycephalic dogs (16 Shih Tzus, 11 English bulldogs, 11 French bulldogs, and 4 Pugs), all of which were adults with ages between 1 and 6 years (mean of $3 \pm 1.26$ years), males $(21.43 \%$ ) or females $(78.57 \%)$ from private kennels. The owners, who signed a consent form, authorized inclusion of these dogs in this study. Degree of kinship was not considered as inclusion or exclusion criteria.

\section{Physical, laboratory and ophthalmic exams}

Physical, laboratory [complete blood count, serum alanine aminotransferase (ALT) and creatinine levels], and ophthalmic examination: direct and consensual pupillary light reflex, menace response, Schirmer tear test (Schirmer Tear Test) ${ }^{1}$, slit lamp biomicroscopy (Slit Lamp Kowa - SL-15L) ${ }^{2}$, applanation tonometry (Tono-Pen $\left.\mathrm{VET}^{\circledR}\right)^{3}$, binocular indirect ophthalmoscopy (Topcon ID-10) ${ }^{4}$, fluorescein dye test (Fluorescein Dye Test) ${ }^{1}$ and tear breakup time test. Dogs that were healthy as assessed in all exams were subjected to ocular ultrasound.

\section{Ophthalmic ultrasound}

Desensitization of the corneas was achieved with proxymetacaine hydrochloride $(5 \mathrm{mg} / \mathrm{ml}$; Anestalcon $\left.{ }^{5}\right)^{5}$ anesthetic drops. Sterile gel (Supragel) ${ }^{6}$ was used as contact medium. The corneal technique was employed using a $20 \mathrm{MHz}$ transducer (Ultrascan) ${ }^{7}$. Dogs were restrained manually in a gentle way.

The imaging device used was an ultrasound scanner (ACUSON S2000) ${ }^{8}$ equipped with a $9.0 \mathrm{MHz}$ transducer. The echogenicity (anechoic, hypoechoic, hyperechoic, or mixed) and echotexture (homogeneous or heterogeneous) of the anterior, posterior, and vitreous chambers, lens, RCSC, and eye fundus (optic nerve) were evaluated.

\section{Contrast-enhanced ocular ultrasonography}

The CEUS exam was performed using contrast specific software (Cadence - Acuson S2000) ${ }^{9}$, with secondary harmonic imaging and inverted pulse technique. After delimitation of the area the probe hold steadily and the adjustable parameters, such as depth, gain, mechanical index and focal zones, were optimized and maintained. Immediately the contrast agent (SonoVue $\AA)^{10}$ was intravenous administered in bolus $(0.1 \mathrm{~mL}$, followed by $5 \mathrm{~mL}$ saline flush) via a catheter placed in the cephalic vein. Images were obtained for five minutes after bolus injection of the contrast agent and recorded on the internal storage system per each assessed tissue.

Videos and images were evaluated according to presence or absence of microbubbles on the optic nerve, RCSC, and ciliary body at wash-in and washout times, as well as at the peak contrast enhancement time. Structures were also classified as hyper, iso, or hypoenhanced, with centripetal or centrifugal filling pattern and according to internal homogeneity, as homogeneous or heterogeneous.

\section{Statistical analysis}

Statistical analysis was performed using software $\left(\mathrm{R}^{\circledR}\right)^{11}$. Normal distribution of data (Shapiro Wilk 
test) and homogeneity of variance (Barlett's test) were checked for the time variables. The comparisons of time variables between right and left eyes, and between genders were performed using the Student's $t$ test. The correlation between time variables and age of animals was also studied (Pearson's correlation). The level of significance was set at $5 \%(P<0.05)$.

\section{RESULTS}

Conventional ultrasound examination did not reveal any alteration in eye structures such as the optic nerve area, RCSC and ciliary body exhibited homogeneous echotexture. Regarding the contrast-enhanced ultrasound technique, no adverse reaction or side effect symptoms related to the intravenous administration of contrast agent were observed in the dogs of this study. The exam was performed without difficulties and contrast filling or perfusion was adequately observed in the structures evaluated.

The RCSC was seen as a single hyperechoic structure that delimits the posterior part of the eye. The optic nerve is seen as a hypoechoic structure located caudally to the optic disk, and the ciliary body is seen as a double, homogeneous, hypoechoic structure located at the periphery of the lens.

Contrast enhancement was observed in 52 out of the 60 eyes evaluated, with a homogeneous filling and centrifugal pattern of distribution. Structures adequately perfused were the optic nerve, RCSC, and ciliary body (Figure 1). The optic nerve region was hyperenhanced (higher intensity of contrast filling) as compared to the other structures, which were similar to each other (isoenhanced).

Regarding evaluation of contrast enhancement time variables, wash-in $(P=0.55)$, peak enhancement $(P=0.26)$, and wash-out $(P=0.54)$ times were similar for right and left eyes, independent of the structure evaluated. Mean values and standard deviation of the time variables observed for the eye structures are described in Table 1. When contrast enhancement parameters were evaluated for each eye structure separately, a shorter wash-in time was seen at the optic nerve region as compared to the other structures, and a longer washin time was seen at the ciliary body; however, peak enhancement time was observed at similar moments. Additionally, the wash-out time was also longer at the optic nerve region (Table 1).

When variables were compared according to the animals' characteristics, no correlation was found between contrast enhancement time and age of animals $(P>0.05)$. However, there were differences between variables in males and females with a longer wash-in time $(P=0.029)$ in males $(5.6 \pm 3.3 \mathrm{~s})$ as compared to females $(3.9 \pm 1.9 \mathrm{~s})$; longer peak enhancement time $(P=0.01)$ in males $(18.4 \pm 4.7 \mathrm{~s})$ as compared to females $(14.8 \pm 3.7 \mathrm{~s})$; and longer wash-out time $(P=$ $0.03)$ in males $(28.8 \pm 3.7 \mathrm{~s})$ as compared to females $(26.2 \pm 3.2 \mathrm{~s})$.

Table 1. Mean values and standard deviation of contrast enhancement time variables (CEUS), wash-in, peak enhancement, and wash-out time at the optic nerve, RCSC, and ciliary body in dogs.

\begin{tabular}{cccc}
\hline \multirow{2}{*}{ Structures } & \multicolumn{3}{c}{ CEUS variables } \\
\cline { 2 - 4 } & Wash-in (s) & Peak enhancement (s) & Wash-out (s) \\
\hline \multicolumn{4}{c}{ Optic nerve } \\
\hline LE & $4.37 \pm 1.74$ & $17.62 \pm 2.92$ & $29.20 \pm 2.02$ \\
RE & $4.13 \pm 1.78$ & $17.75 \pm 3.48$ & $28.82 \pm 1.89$ \\
\hline \multicolumn{4}{c}{ RCSC } \\
\hline LE & $8.06 \pm 1.90$ & $15.10 \pm 1.91$ & $26.03 \pm 1.49$ \\
RE & $7.24 \pm 1.66$ & $14.82 \pm 136$ & $25.68 \pm 1.07$ \\
\hline \multicolumn{4}{c}{ Ciliary body } \\
\hline LE & $8.37 \pm 2.21$ & $16.34 \pm 1.58$ & $26.34 \pm 1.34$ \\
RE & $8.37 \pm 2.41$ & $16.17 \pm 1.51$ & $25.72 \pm 1.70$ \\
\hline
\end{tabular}

CEUS: contrast-enhanced ultrasonography; s: seconds; LE: left eye; RE: right eye; RCSC: retina-choroid-sclera complex. Level of significance: $5 \%$ level of significance. 


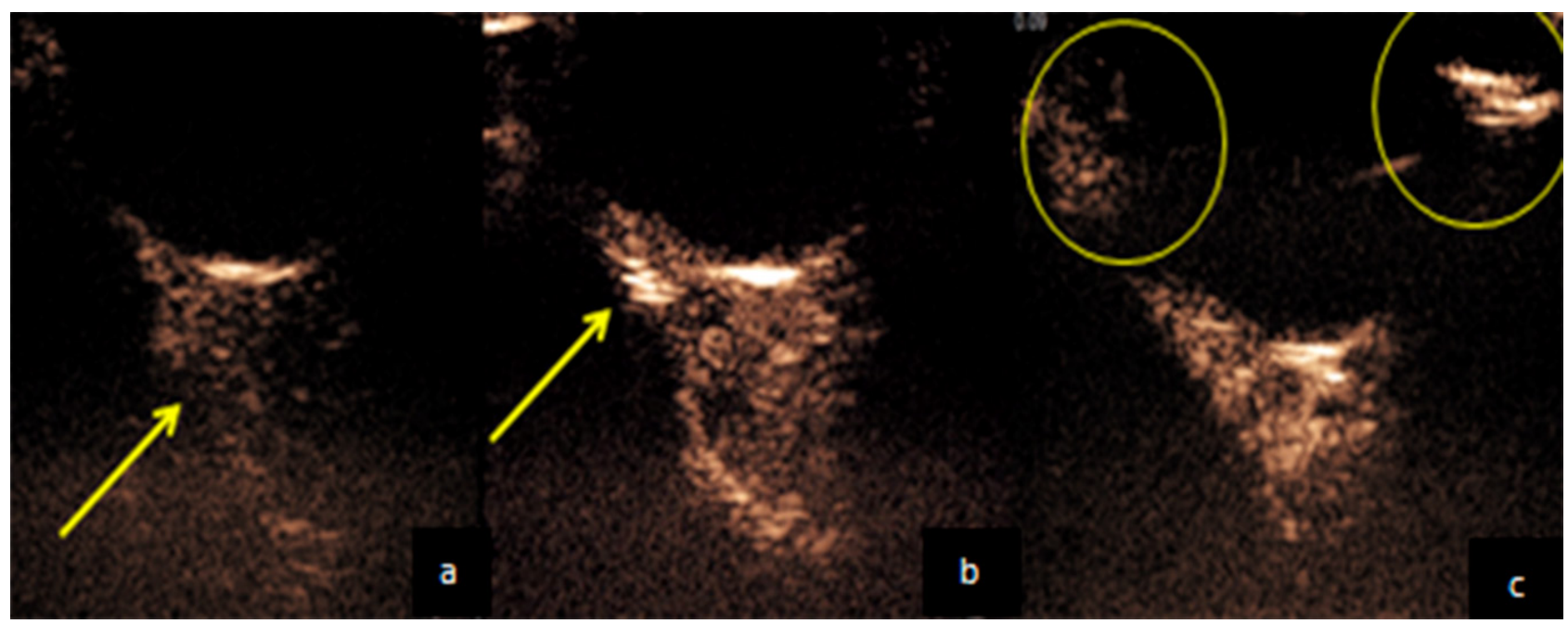

Figure 1. Ultrasound images of the contrast enhancement of the French Bulldog dog's ocular bulb. a- The presence of the contrast in the adjacencies of the optic nerve. b- The filling proceeds to the region of retina-choroid-sclera complex. c- Ciliary body filled with contrast.

\section{DISCUSSION}

Ultrasound is an important diagnostic resource for veterinary ophthalmology and provides relevant information for the detection of pathological conditions of the eyes of animals [4,19].

However, the study of the vascular structures of the eye and abnormalities related to vasculature (vascular characterization and obtainment of vascular indices) requires additional technologies such as Doppler ultrasound [5,7], and, more recently, contrast-enhanced ultrasound. The latter technique allows evaluation of tissues at the capillary level (macro and microcirculation), complementing the vascular ultrasound scan [1]. In this context, this study brings original information on contrast-enhanced ultrasound findings for evaluation of eyes in dogs, creating novel diagnostic possibilities for the use of this imaging technique in veterinary ophthalmology.

Inclusion of imaging techniques that allow adequate study of the vascular features of the eye of dogs, such as Doppler ultrasound, is extremely advantageous for veterinary routine, since it is not invasive and do not exhibit any deleterious effect for the patient, allowing diagnosis of hemodynamic dysfunction and vascular abnormalities in several tissues [7]. Similarly, the contrast-enhanced ultrasound technique used in this study was demonstrated to be effective for the evaluation of the vascularization of eyes of dogs, with absence of side effects and no need for chemical restraint (sedation or anesthesia) of patients.
Microbubble contrast media enable evaluation of the vascularization of the tissues analyzed in this study in detail, especially because there is potentialization of ultrasound echoes [9]. The study of the macrocirculation allows visualization of vascular flow as well as morphology of the vessel's lumen, and assessment of perfusion allows characterization of pattern and intensity of tissue uptake, which are especially useful in cases of neoplastic lesions [3]. The importance of the present study is based on the lack of reports on the evaluation of the vascular pattern of eyes of dogs using microbubble contrast.

The vascular pattern identified in this study, with enhancement starting at the region of the optic nerve and retina, and then ciliary body (with the optic nerve as the most hyperenhanced structure) is consistent with the expected pattern for dogs without ophthalmic disorders. The posterior ciliary artery is primarily responsible for supplying the optic nerve, later irrigating the retina, lateral and medial segments of the ciliary body [13].

Some works on the evaluation of orbital vascular pattern in humans report a significant variability on anatomical course of the ophthalmic artery and its branches [13]. However, in our study, no significant variation was observed on vascular flow of dogs of different brachycephalic breeds.

Retinal blood vessels consist of arteries and veins that emerge from the optic nerve region and from the choroid. Contrast enhancement at the region of the 
RCSC is justified by the presence of vessels from the choroid that are irrigated by posterior ciliary arteries and drained by vorticose veins, since this structure is responsible for the nutrition of the external portion of the retina [6].

Branching of the main artery explains the centrifugal vascular pattern observed. The central branches of the retina irrigate the eye fundus, and the short posterior ciliary arteries supply the choroid and external portion of the retina. The long posterior ciliary arteries reach the ciliary body and iris, while anterior ciliary arteries irrigate sclera, episclera, limbus, bulbar conjunctiva, and ciliary plexus. Drainage occurs through superior and inferior ophthalmic veins [15].

Wash-in, peak enhancement, and wash-out times were similar for right and left eyes owing to anatomical and physiological similarities in vascularization [15]. The homogeneous contrast-filling pattern is of paramount importance, since it will allow differentiation of abnormalities in sick animals, such as partial or total loss of capillarization, which will cause heterogeneity or absence of capillary fill. It will also be important for early diagnosis and evaluation of vascular alterations, especially ischemic changes, especially glaucoma, intraocular neoplasm, progressive retina atrophy, and uveitis.

The age did not interfere with vascular pattern of the eye of dogs, which were similar in young adults of different ages. These data demonstrate the strength and integrity of capillaries in the eyes of dogs; however, more studies are necessary to include a larger number of dogs distributed into different age groups in order to allow the evaluation of differences between breeds, weights, gender, and age that could interfere in the vascular pattern. Knowledge on the circulatory pattern of the eye fundus of these patients may greatly improve diagnosis and prognosis prediction in cases of diabetic retinopathy and/or glaucoma, in diseases that affect the retinal vasculature [10], and in cases that require evaluation of neovascularization resulting from neoplastic processes, especially in the eye fundus.

In medicine, ultrasound techniques have helped evaluation of retinal vascular compromise in diabetic patients [18]. In considering the efficacy of the microbubble contrast method for the evaluation of the retinachoroid-sclera complex observed in the present study, it is possible that this technique will help monitoring patients with diabetic retinopathy.

Doppler ultrasound has proven useful for the evaluation of systemic diseases such as toxoplasmosis, viral feline immunodeficiency, cancers, and fungal diseases, all of which may interfere with arterial vascularization of the eye in felines [21]. Since, in the present study, the microbubble contrast method proved effective to evaluate vascularization of eye structures in dogs, it is presumable that ophthalmic repercussions of systemic diseases in dogs may be better investigated using this technique.

\section{CONCLUSION}

We conclude that ophthalmic ultrasound evaluation using microbubble contrast provides relevant information regarding the vascular pattern of brachycephalic dogs. This exam was fast, non-invasive, and free from any adverse effects related to intravenous infusion of the contrast. The present work validates the use of the microbubble contrast technique for the sonographic study of the ophthalmic vascular pattern in dogs, and, consequently, may be useful as a reference for the evaluation of ophthalmic vascular alterations secondary to ophthalmic or systemic diseases in this species.

\section{MANUFACTURERS}

${ }^{1}$ Ophthalmos S.A. Sao Paulo, SP, Brazil.

${ }^{2}$ Kowa Company Ltd. Tokyo, Japan.

${ }^{3}$ Reichert Technologies Inc. Depew, NY, USA.

${ }^{4}$ Topcon Great Britain Ltd. Newbury Berkshire, UK.

${ }^{5}$ Alcon Laboratórios do Brasil Ltda. São Paulo, SP, Brazil.

${ }^{6}$ Adlin Plásticos Ltda. Jaraguá do Sul, SC, Brazil.

${ }^{7}$ Alcon Laboratories. Fort Worth, TX, USA.

${ }^{8}$ Siemens Medical Solutions Inc. Malvern, PA, USA.

${ }^{9}$ Siemens. Munich, Germany.

${ }^{10}$ Bracco Corporate. Milan, Lombardy, Italy.

${ }^{11} \mathrm{R}$ Foundation for Statistical Computing. Vienna, Austria.

Acknowledgements. The authors would like to thank FAPESP for the financial support (processes 2012/16635-2 e 2013/06443-1).

Ethical approval. The trial was conducted following approval by local animal use committee (UNESP Jaboticabal) (protocol number 9516/15).

Declaration of interest. The authors report no conflicts of interest. The authors alone are responsible for the content and writing of the paper. 


\section{REFERENCES}

1 Brito M.B.S., Feliciano M.A.R., Coutinho L.N., Uscategui R.R., Simões A., Maronezi M.C., de Almeida V.T., Crivelaro R.M., Gasser B., Pavan L. \& Vicente W.R.R. 2015. Doppler and Contrast-Enhanced Ultrasonography of Testicles in Adult Domestic Felines. Reproduction in Domestic Animals. 50: 730-734.

2 Carvalho C.F., Chammas M.C., Cogliati B., Carrilho F.J. \& Cerri G.G. 2013. Elastography and contrast-enhanced ultrasonography improves early detection of hepatocellular carcinoma in experimental model of NASH. Journal of Clinical Experimental Hepatology. 3: 96-101.

3 Chammas M.C. 2009. Ultrassonografia abdominal. 2.ed. Rio de Janeiro: Revinter, 832p.

4 Dietrich C.F. \& Cantisani V. 2014. Current status and perspectives of elastography. European Journal of Radiology. 83: 403-404.

5 Diniz A.L.D., Moron A.F., Santos M.C., Moron A.F. \& Sass N. 2004. Dopplervelocimetria colorida dos vasos: técnica de exame e anatomia vascular normal. Radiologia Brasileira. 37: 287-290.

6 Dyce K.M., Sack W.O. \& Wensing C.J.G. 1997. Tratado de Anatomia Veterinária. 2.ed. Rio de Janeiro: Guanabara Koogan, 663p.

7 Feliciano M.A.R., Abrahim M.A., Peixoto R.V.R., Yasunaga K. L., Vicente W.R.R. \& Galera P.D. 2013. Contribution of ocular B-mode and triplex Doppler in the evaluation of Poodle dogs with cataracts. Arquivo Brasileiro de Medicina Veterinária e Zootecnia. 65: 359-363.

8 Fischer T., Dieckhofer J., Muhler M., Lembcke A., Morgera S., Budde K., Neumayer H. H., Ebeling V., Thomas A. \& Filimonou S. 2005. The use of contrast-enhanced US in renal transplant: first results and potential clinical benefit. European Journal of Radiology. 15: 109-116.

9 Goldberg B.B., Liu J.B. \& Forsberg F. 1994. Ultrasound contrast agents: a review. Ultrasound in Medicine and Biology. 20: 319-333.

10 Gonçalves G.F., Pippi N.L., Leme M.C., Custódio A.T., Silva A.V., Romagnolli P., Pachaly J.R., Bottin J.M.P. \& Livero F.A.R. 2008. Correlação entre a pressão arterial média e o fluxo sanguíneo na artéria oftalmica externa em gatos (Felis catus Linnaeus, 1758). Brazilian Journal of Veterinary Research Animal Science. 45: 57-66.

11 Haers H., Vignoli M., Paes G., Rossi F., Taeymans O., Daminet S. \& Saounders J.H. 2010. Contrast harmonic ultrasonographic appearance of focal space-occupying renal lesions. Veteterinary Radiology Ultrasound. 51: 516-522.

12 Halpern E.J., Frauscher F., Rosenberg M. \& Gomella L.G. 2002. Direct biopsy during contrast-enhanced sonography of the prostate. American Journal of Roentgenology. 178: 915-919.

13 Hayreh S.S. 2004. Posterior Ciliary Artery Circulation in Health and Disease The Weisenfeld Lecture. Investigative Ophthalmology \& Visual Science. 45: 749-757.

14 Hom B.K., Shrestha R., Palmer S.L., Katz M.D., Selby R.R., Asatryan Z., Wells J.K. \& Grant E.G. 2006. Prospective evaluation of vascular complications after liver transplantation: comparison of conventional and microbubble constrast enhanced US. Radiologia Brasileira. 241: 267-274.

15 Junqueira L.C. \& Carneiro J. 2004. Histologia Básica. 10.ed. Rio de Janeiro: Guanabara Koogan, 118p.

16 Kalantarinia K. \& Okusa M.D. 2007. Ultrasound contrast agents in the study of kidney function in health and disease. Drug Discovery Today: Disease Mechanisms. 4: 153-158.

17 Kang D.W., Lee S.C., Park Y.G. \& Chang J.H. 2012. Long-term results of gamma knife surgery for uveal melanomas. Journal of Neurosurgery. 117: 108.

18 Karami M., Janghorbani M., Dehghani A., Khaksar K. \& Kaviani A. 2012. Orbital Doppler Evaluation of Blood Flow Velocities in Patients with Diabetic Retinopathy. The Review of Diabetics Studies. 9: 104-111.

19 Kendal C.J., Prager T.C., Cheng H., Gombos D., Tang R.A. \& Schiffman J.S. 2015. Diagnostic Ophthalmic U1trasound for Radiologists. Neuroimaging Clinics of North America. 25: 327-365.

20 Maronezi M.C., Feliciano M.A.R., Crivellenti L.Z., Botin-Crivellenti S., Silva P.E.S., Zampolo C., Pavan L., Gasser B., Simões A.P.R., Maciel G.S., Canola J.C. \& Vicente W.R.R. 2015. Spleen evaluation using contrast enhanced ultrasonography and Doppler in dogs with subclinical ehrlichiosis. Arquivo Brasileiro de Medicina Veterinária e Zootecnia. 67: 1528-1532.

21 Mattoon J.S. \& Nyland T.G. 2002. Small Animal Diagnostic Ultrasound. 2nd edn. Philadelphia: Saunders, 461p.

22 Nilsson A. 2004. Contrast-enhanced ultrasound of the kidneys. European Radiology. 14: 104-109. 
23 Nogueira A.C., Morcerf F., Moraes A.V. \& Dohmann H.F.R. 2002. Ultrasonografia com agentes de contrastes por microbolhas na avaliação da perfusão renal em indivíduos normais. Revista Brasileira de Ecocardiografia. 15: 74-78.

24 Vanderperren K., Haers H., Van der Vekens E., Stock E., Paepe D., Daminet S. \& Saunders J.H. 2013. Description of the use of contrast enhanced ultrasonography in four dogs with pancreatic tumours. Journal of Small Animal Practice. 55: 164-169.

25 Venturini M., Colantoni C., Modorati G. Di Nicola M., Calucci A., Agostini G., Picozzi P., De Cobelli F., Parmiani G., Mortini P., Bandello F. \& Del Maschio A. 2015. Preliminary Results of Contrast-Enhanced Sonography in the Evaluation of the Response of Uveal Melanoma to Gamma-Knife Radiosurgery. Journal of Clinical Ultrasound. 43: 421-430.

26 Vignoli M., Russo M., Catone G., Rossi F., Attanasi G., Terragni R., Saunders J. \& England G. 2011. Assessment of vascular perfusion kinetics using contrast-enhanced ultrasound for the diagnosis of prostatic disease in dogs. Reproduction on Domestic Animals. 46: 209-213.

27 Xu Q.H., Zhao C., Zhu J.G., Chen M.J. \& Liu Q.H. 2015. Helium-neon laser therapy in the treatment of hydroxyapatite orbital implant exposure: A superior option. Experimental and Therapeutic Medicine. 10: 1074-1078.

28 Yang W.L., Wei W.B. \& Li D.J. 2012. Quantitative parameter character of choroidal melanoma in contrast enhanced ultrasound. Chinese Medical Journal. 125: 4440.

29 Yuan J.Y., Zhang J.H., Tang C., Zhu H., Xie H. \& Gao S.J. 2011. Application of ultrasound microbubble contrast technology in ophthalmic targeted therapy: literature analyses. International Journal of Ophthalmology. 4: 537-542. 